\title{
Johann Falkenbergs Stellung zur Papstfrage in der Zeit vor dem Pisaner Konzil (1408).
}

\author{
Von
}

\section{Gustav Sommerfeldt.}

Über Falkenbergs Abhandlung „De renuntiatione papae" war von mir in Zeitschrift für die Geschichte des Oberrheins 18, 1903, Seite 418 Anmerkung die Vermutung aufgestellt worden, es könne diese statt. zu 1408 vielmehr zum Jahre 1417 zu setzen, und also dem Konstanzer, nicht dem Pisaner Konzil zuzurechnen sein.

Indem jedoch durch die neuerdings erfolgte Veröffentlichung des Handschriftenkatalogs der Prager Universitätsbibliothek den daselbst befindlichen Beständen eine genauere Bekanntgabe zuteil geworden ist, stellte sich heraus, dass in dieser Bibliothek der Traktat De renuntiatione papae nicht nur in der undatierten, aber zu etwa 1420 anzusetzenden Handschrift 1878 (= XC 25), Blatt 267a--270a vorliegt, wonach Höfler und von Schulte auf ihn bezug nabmen'1), sondern ausserdem noch in den zwei Handschriften 1889, Blatt 104 a-106 a und 1996, Blatt $91 \mathrm{a}-93 \mathrm{~b}$, von denen die letztere den Traktat mit

1) K. Höfler, Ruprecht von der Pfala, genannt Clem, römischer König, 1400-1410. Freiburg 1861. S. 411 and 420, wo der Verfasser jedoch Walkernberg genannt wird; J. F. v. Schulte, Geschichte der Quellen und Literatur des kanonischen Rechts. Bd. II. Stuttgart 1877. S. 381. - Blatt 246 ff. in dem Prager Kodex 1878, ist von älterer Hand des Matthäus von Krakau Schrift „De praxi curiae Romanae“, nachmals De squaloribus genannt, anzutreffen. Vergl. G. Sommerfeldt in Z. für die Geschichte des Oberrheins 18, S. 431-432. 
Datierung rom 7. Dezerber 1408 (proxima feria sexta post festum sancti Nicolai) aufweist ${ }^{1}$ ). Auch hat derselben Handschrift zufolge - 1878 und 1889 sind Abschriften aus ihr - Falkenberg diesen Traktat zu Prag als These wissenschaftlich verteidigt. Es hat sich der Dominikaner nach Prag jedenfalls von Krakau aus begeben, nachdem die Vorgänge des Jahres 1406, speziell das Prozessverfahren, das der Wormser Bischof Matthäus von Krakau in Rom gegen Falkenberg wegen dessen die angebliche Häresie des Matthäus betreffender Angriffe hatte einleiten lassen, ein längeres Verweilen Falkenbergs zu Krakau ihm und dem Konvent seines Ordens als gefährlich erscheinen lassen musste. Die letzte urkundliche Erwähnung, die uns Falkenberg in Krakau zeigt, datiert rom Juni $1406^{2}$ ).

Was die Jugend und den Bildungsgang Falkenbergs anlangt, so liegen darüber bedauerlicher Weise wenig positive Zeugnisse vor. Nur soviel steht fest, dass Falkenberg ein Norddeutscher war, seine Bildung im Dominikanerkloster Kammin des Herzogtums Pommern erbielt $^{3}$ ), und am 18. März 1381 in der Artistenfakultät zu Prag die Lizentiaten würde erlangte ${ }^{4}$ ).

Er hat diese Universität dann aus Anlass des im Jahre 1384 stattgefundenen Kollegiaturenstreits verlassen und ist nach Wien übergesiedelt, wo er im Sommersemester 1385 als „Magister Johannes Valkenberch Saxo" sich immatrikulieren liess ${ }^{5}$ ). Der Zusatz zum :S. 1997.

1) J. 'Truhlár̆, Catalogus codicum manuscriptorum Bd. I. Prag 1905.

2) G. Sommerfeldt, im Historischen Jahrbuch (München) 27, 1906, S. 612; M.Ritter in Allgem. deutsche Biographie 6, S. 554; B. Bess, in Zeitscbrift für Kirchengcschichte 16, S. 385; J. Fijalek, Mistrz Jakób z Paradjża Bd. I. Krakau 1900. S. 5.

s) J. Quétif und J. Echard, Scriptores ordinis praedicatorum. Bd. I. Paris 1719. S. 760; K. Morawski, Historya uniwersytetu Jagiellonskiego. Bd. 1. Krakau 1900. S. 150: J. Caro, Geschichte Polens. Bd. IlI. S. 464. Über das Kloster zu Kammin, dessen Prior um 1380 Alexander biess, siehe L. Kü cken, Geschichte der Stadt Kammin, in Pommern. Kammin 1880; J. J. Steinbrück, Geschichte der Klöster in Pommern und den angrenzenden Provinzen. Stettin 1796. S. 24-27.

4) Monumenta historiae universitatis Pragensis. Bd. I. Prag 1830. S. 197, vgl. S. 212.

5) Die Angabe bei J. Aschbach, Geschichte der Wiener Universität. Bd. I. Wien 1865. S. 114 und 613, dass die Bezeichnung in den Acta facultatis artistarum I Folio 25 a gelautet habe "Johannes de Valkenberg", ist ungenau. Bei R. K ink, Geschichte der Universität Wien. Bd. I, 2. Wien 1854. S. 9 ist er nach eben jener Vorlage richtiger genannt: Magister Johannes Vulkchenberch. 
Namen wollte wahrscheinlich darauf hinweisen, dass Falkenleurg in Prag der Sächsischen Nation der Artistenfakultät angehört hatte. Man rechnete dieser Nation in Prag den grössten Teil Norddeutschlands zu. Unterm 13. Noveuber 1385 wird Falkenberg au vierter Stelle unter den Examinatoren genannt, die zu Prüfung der Bakkalaren der Wiener Artistenfakultät ernannt wurden ${ }^{1}$ ). Im Laufe des Jahres 1386 hat Falkenberg dann vermutlich Wien verlissen, denn bei der Ende Februar 1387 stattfindenden Beeidigung der Magister der Artisteufakultät ist sein Name nicht mehr anzutreffen ${ }^{2}$ ).

Die Beneunung seiner Fumilie geht eventuell auf eine der kleinen Ortschaften, deren mehrere in Pommern sich vorfinden ${ }^{3}$ ), zurück, dagegen beruht die durch eine Notiz Quétifs*) veranlasste Meinung der Historiker, dass der Dominikaner seinen Namen nach der in der Drage in Hinterpommern (im heutigen Kreise Dramburg) belegenen Stadt Falkenburg habe ${ }^{5}$ ), sicherlich auf einer Ferwechslung der Familie Falkenbergs mit dem bekannteren, aus dem Schlosse der Stadt herstammendeu Kittergeschlecht derer von Falkenburg. Annehmbarer als die Meinung Quétifs ist Fabricius Vermutung. dass Falkenberg „aus Preussen oder Livland" gebürtig sei6). Wir werden in dieser Hinsicht durch den Umstaud jedenfalls bestärkt, dass ein „Johaunes Falkenborg de Danczk" (also aus Danzig), sich Ostern 1405 in die Matrikel der Uuiversität Erfurt eintragen liess ${ }^{2}$ ), und dass der Dominikanerpater Magister Johaun Falkenberg in einer vom 18. November (feria IV. post Martini) 1411 datierten „Determinatio" 8, die er zu Magdeburg verteidigte, sich bezeichnet hat als „Inquisitor haereticae pravitatis per Saxonian et Thuringiam".

1) Wien, Universitatsarchiv, Acta facultatis 1 Folio 1?0.

9) Aschbach I, S. 120 .

3) Drei im heutigen Kreis Pyritz, je eine in den Krejsen Naugard und Schivelbein. Vgl. Ste in brü ck, S. 16 und 43, ferner Pommerisches Urkundenbuch Bd. I. Stettin 1877. S. 33, 8j, 177 und öfter, II, S. 471 und 504, III, S. 231 .

1) Quétif und bicbard $\$ .760$.

5) M. Wiszniewski, Historya literatury polskiej. Bd. ILL. Krakau 1841 . S. 134; A. Knöpfler, in Wetzer und Welte's Kirchenlexikon 6, Spalte 1660; Bess a. a. O. S. 392: Morawski I, S. 150 .

6) J. A. Fabricius, Bibliotheca latina mediae aetatie. Bd. IV. Hamburg 1735. S. 211. - v. Schulte, II, S. 381 nimnt ohne weiteres Pommern als die Heimat Falkenbergs an.

7) J. C. H. We issenborn, tkten der Erfurter Universität. Bd. I. Halle 1881. S. 74 .

8) Wien, Hof bibliothek Kodex Lat. 4902 , Blatt 93 a-96a; vgl. v. Schulte JI, S. 382, Anm. 3. 
Die heftigen Angriffe in dem um wenige Jahre älteren Traktat. „De renuntiatione papae gegen Gregor XII., den unser Magister des. Meineids, des Wortbruchs und der Häresie zeiht ${ }^{1}$ ), müssen umsomehr in Erstannen setzen, da Falkenberg 1406 eben diesem Papst von. Krakau aus die Schrift De monarchia mundi unter Ausdrücken tiefster Devotion zugeeignet hatte ${ }^{2}$ ).

Wenn Höfler a. a. O. S. 420 der Annahme Raum gibt, dass die Stellongnahme Falkenbergs im Traktat des Jahres 1408 mit der veränderten Politik zusammenhängt, die König Wenzel damals anfing zu befolgen ${ }^{3}$ ), so kann dem mangels näherer Beweise ohne weiteres gewiss nicht beigepflichtet werdeu. Es ist vielmehr das Bekanntwerden der im Herbst 1407 zu Siena durch den Erzbischof von Ragusa, Nicolaus de Sacchis, Dominikaner und gewesenen Theologieprofessor, zu Gunsten Gregors XII. veröffentlichten Abhandlung4), daneben der Falkenberg.

1) r. Schulte, II, S. 382; L. Pastor, Geschichte der Päpste. Bd. I. Freiburg 1886. S. 144. Ailli und die französische Schule nahmen in der Frage etwaiger Häresie der Päpste einen weit weniger extremen Standpankt ein als Falkenberg; P. Tschackert, Peter von Ailli. Gotha 1877. S. 128. Über das Absetzungsrecht gegenüber den Päpsten hatte Falkenberg auch in Teil IV. Kapitel 1 seiner Schrift De monarchia mundi gehandelt.

2) Historisches Jahrbuch 27, S. 609-610. Nach Prenssen, wo er iw Auftrage des Deutschritterordens seine Schrift gegen König Wladislaw II. Jagiello verfasste (J. Lenfant, Histoire du concile de Pise. Bd. IL. Amsterdam 1727. S. 211-212; 248-250 u. 273; Caro III, S. 465 ff. Morawski I, S. 150-151), kann Falkenberg frühestens 1409 sich begeben haben. Die Schicksale während seiner nicht allzu strengen Haft bei der Kurie in deu Jahren 1418 bis 1424 ergeben sich aus J. Dlugoss' bei Lenfant II, S. 249 verwerteten Erzäblung und aus H. V.Sauerlands Regestenbeitrag in Altpreussische Monatsschrift 46, 1909, S. 49-57. Vgl. auch Bess S. 392 und 445 ff. und A. Lewicki, Codex epistolaris saeculi 15. Bd. II (Monumenta medii aevi historica Bd. XII). Krakau. 1891. S. 113 u. $170-174$. Bd. II.

3) Th. Lindner, Geschichte des deutschen Reichs unter König Wenzel

4) Von den drei Schriften, in denen Nikolaus über die Zessionsfrage und das Verhalten Gregors handelte, - Falkenberg scheint nur die erste davon vorgelegen zu haben - spricht Dietrich von Niem in seinem Hauptwerk, De schismate, ed. S. Schard. Basel 1566 S. 178 . Die obige, sicher vom Jahre 1407 herstammende ist ohne nähere Datierung gedruckt in Dietrichs, Nemus unionis ‘, ed. Schard ebd. Seite 234-240, unter dem Titel, Quaestio super renunciacionem papatus a Gregorio XII. vel faciendam vel non faciendam G. Erler, Die historischen Schriften Dietrichs von Niem. Leipzig 1887. \$. 22, der mit Recht gegen Lenz hervorhebt, dass nur Nikolaus de Sacchis gemeint sein kann, nicht sein Nachfolger Johann Dominici, der das Erzbistun Ragusa. am 26. Mărz 1408 erhielt. Erzbischof Nikolaus starb am 13. Januar 1408, vgl. P. Gams, Series episcoporum S.414, wo er unrichtig als Andreas de Sacchis. 
innewohnende, und trotz der gemachten üblen Erfahrangen nicht einzuschränkende Trieb, in den das kirchliche Gebiet betreffenden Tagesfragen unmittelbar Partei zn ergreifen, die Veranlassung zum Entstehen der trotz hie und da vorhandener scharfer Ausfälle doch einigermassen seichten, zu höheren Gesichtspunkten sich kauı irgendwo erhebenden "Quaestio“ Falkenbergs über die Abdankung des Papstes gewesen. - Aus jenem Drange luerans, weit weniger als Ergebnis des Interesses, das Falkenberg an dem Deutschritterorden nahm, ist auch der auf dem Konstanzer Konzil später zum Ausbruch gekommene Streit mit Paul Wladimiri zu erklären. Die von verstärkten Angriffen gegen den Polenkönig und dessen Familie erfüllte, inhaltlich zudem etwas an De monarchia mundi sich anlehnende Schrift, De doctrina potestatis papae et imperatoris" ${ }^{1}$ ) ist aus diesem Streit herrorgegangen. Motive änsserlicher und persönlicher Art waren es nicht minder, die Falkenbergs Erwiderungsschrift (1416) auf die Abhandlung hervorriefen, die Pierre d'Ailli gegen die den Tyrannenmord betreffenden Thesen des Pariser Professors Jean Petit veröffentlicht hattea). Ond demselben unbezähmbaren Streben Falkenbergs zu oppositionellem Auftreten entsprang der Konflikt, in den er sich sogar mit dem eigenen Ordensgeneral Leonardus de Datis verwickelte ${ }^{3}$ ).

Was die gegenwärtig uns interessierende "Quaestio * vom Jahre 1408 anlangt, so hat diese ausser in den drei Prager Kodices sich auch in einer Abschrift zu Wien, Hof bibliothek Cod. Lat. 4754, Blatt $128 \mathrm{~b}$ $138 \mathrm{~b}$, und in einer undatierten, aber bald uach 1410 hergestellten

bezeichnet ist. Vgl. auch A. Rösler, Kardinal Johannes Dominici, 1357 bis 1419, ein Reformbild aus der Zeit des grossen Schismas, Freiburg 1893. S. 154. Dietrich von Niem a. a. O.S. 178 gibt an, dass Nikolaus de Sacchis Professor der Theologie (in sacra pagina) gewesen sei, Ende September 1407 bei der Kurie (cirea festum Michaelis) zu Siena sich authielt, dann mit dieser nach Lucca sich begab, und am 13. Jănner 1408 mitten in seinen weiteren literarischen Entwürfen eines plötzlichen Todes verstarb. Über das Verhalten des Dominikanerordens un diese Zeit $\nabla g l$. Wiszniewski III, S. 135. - Dass Nikolaus obige Abhandlang in den Spătherbst 1407 gehört, ergibt sich namentlich auch daraus, dass das in Dietrichs Nemus unionis folgende Stūck (S. 240 bis 247) rom 1. November 1407 datiert ist.

1) Nach der Handschrift 166 des Ossolińskischen Instituts zu Lemberg gedruckt bei M. Bobrzy ński, Starodawne prawa polskiego pomniki. Bd. V, 1. Krakau 1877. S. 192-232, vgl. auch Bess S. 420 .

2) Lenfant a. a. O. II, S. 212 u. 248; T s chackert S. 244.

s) v. Schulte II, S. 382; Morawaki I, S. 156-157. - L. E. Du Pin in Gersonii opera Bd. V. Antwerpen 1706, Spalte 1013, fasst sein ebenfalls abfälliges Urteil über Falkenberg in die Worte zusammen: Iste erat Jacobita, ut patet in manuali magistri Jacobi de Christo, notarii in causis fidei. 
$K^{K o p i e}{ }^{1}$ ) vorgefuuden in Eichstätt, Königl. Bibliothek Nr. 698 (ältere Zählung 269) Seite 338-344. Obgleich diese Kopie in der zweiten Hälfte eine grosse Lücke des Textes aufweist, und auch fehlerbafte Lesarten gegenüber der besseren Handschrift vielfach anzutreffen sind, hat doch an einzelnen Stellen $E$ zur Jìrgänzung nicht gainz vollständiger Satzwendungeu der Handschrift $P$ herangezogen werden können.

Die nachstehende, auf den vier bezeichneten Handschriften biasierende Wiedergabe des in 7 Konklusionen und 10 Gegenkonklusionen eingeteilten Traktats mag sich aus dem Interesse rechtfertigen, das der Kundgebung des Parteimannes Falkenberg trotz der Einseitigkeit des Standpunkts für jene erregten, das Pisaner Konzil vorbereitenden Zeitläufte innewohnt:

"Tractatas magistri Johannis Valkenberg, ordinis predicatorum, professoris sacre theologie, de renunciacione pape*2).

\Ad pauca respicientes, pocius tamen ad placita quam vera, de facili enuncciant, quod papa semper potest ad placitum libere papatai renuncciare, aliis asserentibus oppositum. quod via ${ }^{3}$ ) renuncciacionis est dyabolica et dampnata. Ideo net) in erroris caligine amplius divine legis ignaros detineant $^{5}$ ), per medium illorum volo ire et ostendere, quomodo papa et quando potest aut debet papatui renuncciare, et innitendo $0^{6}$ ) proprietatem sermonis videtar michi primo $0^{7}$ ), et absque formidine assero, quod papa potest libere papatui renuncciare. Nam, ut ait Anshelmus ${ }^{8}$ ) capitulo 12 de casu dyaboli: homo dicitur posse aliquid potestate, non que in ipso, sed que in altero est, sicut liber potest scribi potestate, non que in libro, sed que in scriptore est. Sed potestate, que in deo est, papa potest liber, papatui renuncciare, quia deus potest sibi precipere aut revelare, quod $^{9}$ ) papatui libere renuncciet10), sicut Cyriaco pape divinitus revelatum legitur, quod relicto papata sacro choro virginum ${ }^{11}$ ) se sociaret, palmam martirii

j) Seite 398-402 sind in dem Kodex mebrere Briefe des Jahtes $1+10$ von derselben Hand, die den Traktat Falkenbergs gegeben hat, zur Niederschrift ge langt. Der Traktat ist hier nicht ganz vollstäudig, auch weist die Abschrift eine grosse Zahl von Fehlern auf.

2) P (1878), Blatt 267 a und P' (1996), Blatt 91 a :, Incipit tractatus magisti Johannis Walkemberg ( $\mathrm{Pl}^{1}$ : Valkemberg), ordinis predicatorum, professoris sacr: theologie, de renuncciacione papes. - E (Eichstätt) Seite 338: , Incipit tractatıs magistri Johannis Valkenberck, ordinis predicatortum, professoris theologie, de renuncciacione pape etc`.

s) P: quod. quia. - i) E: nomini me. - s) $\mathrm{Pl}^{\text {: }}$ detineat. ... 9) $\mathrm{P}$ : intuends. - 7) L (am Rande): Prima conclusio, $\mathrm{P}^{1}$ : innuit conclusionem primam. - $\left.{ }^{8}\right) \mathrm{PP}^{\mathrm{i}}$ : Anzhelnuus. -- ") quod om E. - 10) E: renuncciare.

11) E: sacro numeo et cetni virginum. - Über die um das Jahr 238 spielend", jedoch erst in Chroniken dea 13. Jahrhunderts auftauchende Legende vom Pap:t Cyriacus siche J. Döllinger, Pupstfabeln, München $1863 \mathrm{~S}$. 4ว ff. 
percepturus. Et ergo absque formidine assero et absolute concedendum video ${ }^{1}$ ), quod papa potest libere papatui renancciare, nt recte ait decretalinm de renuncciacione libro 6, capitulo 1 , ponens ${ }^{2}$ ), quod Romanus pontifex, cum se insufficientem agnoscat ad regendum universalem ecclesiam, renuncciare valeat papatui et libere resignare. Et quia papa deo ipsum confortante non insufficiens, sed sufficiens est ad regendum universalem ecclesiam - omnia, inquit apsstolus, possum in eo, qui me confortat, ad Philippenses ${ }^{3}$ ) 一, ideo licet papa, quando illuminatus se insufficientem agnoscit ad regendum universalem ecclesiam, poterit libere resignare. Non tamen potest libere resignare ideo, quia per virtatem propriam se insufficientem agnoscit ad regendum universalem ecclesiam, eo quod forte proponitur ${ }^{4}$ ): quandoque autem ${ }^{5}$ ) possibile est, quod per virtutem propriam se insufficientem agnoscit ad regendum universalem ecclesiam, et tamen deus vult eum regere universalem ecclesiam, et ${ }^{6}$ ) ad regendum confortare. Unde Exodi $4^{7}$ ), cum Moyses dixiseet, obsecro, domine, non sum eloquens $\mathrm{ab}$ heri $^{8}$ ) et nudius tercius, dominus respondit ad enm ${ }^{9}$ ): ego ero in ore tuo doceboque te, quid loquaris. Et ideo sequitur conclusio secnnda, et pono, quod papa non potest sine licencia dei papatui renuncciare. Nam papa contra voluntatem et intencionem dei committentis facit, quando sine eias licencia renuncciat papatni. Sed nemini licet ${ }^{10}$ ) facere scienter contra intencionem ${ }^{11}$ ) dei et roluntatem, et ergo papa non potest renuncciare sine licencia dei papatui. Patet, quia voluntas et intencio dei conmittentis papatum, est, ut exequatur ${ }^{12}$ ), secundum quod potest, papa illud, quod sibi ab eo conmittitur. Et ergo papa, si absque licencia dei renuncciet papatai, non exequitur ${ }^{12}$ ), secundum quod potest, illud, quod sibi $a b$ eo $^{13}$ ) conmittitur. Et jdeo contra eius facit voluntatem et intencionem. Secundo Petrus, quia a deo sibi preceptive dictum est: pasce oves meas, Johannis $21^{14}$ ), non potuit renancciare papatui sine licencia dei. Sed sicat Petrus tanc, ita papa et nunc inmediate a deo recepit curam pastoralem, quia potestas nostra non est ex homine, sed ex deo, in quid ${ }^{15}$ ) papa de indiciis novit. Et16) sancta Romana ecclesia katholica et apostolica nullis synodicis ${ }^{17}$ ) constitutis ceteris ecclesiis prelata ${ }^{18}$ ) est, sed evangelica voce domini et salvatoris nostri primatum obtinait, distinccione 21 , quamvis, et distinccione 22, sacrosancta; et ergo nec pipa potest nunc papatui sine licencia dei renuncciare. Forte dicis, quod Christus evangelica voce Petro sine honine medio papatum conmisit, et nunc pape mediante homine, videlicet collegio cardinalium, et ergo licet Petras non potuit renuncciare papatui sine licencia dei, papa tamen semper libere ad nutum potest papatui renumcciare. Respondeo et dico, quod suppositum, quanto est prius in agendo, tanto virtus eius est inmediacior effectui, quia ${ }^{19}$ ) virtus cause prime coninngit causam secundam suo effectui ${ }^{20}$ ). Unde et prima principia dicantur inmediata in demonstrativis scienciis. Licet ergo cardinales in eleccione pape

1) E: videtur. -- 2) P: dicens. - 9) Philipp. 4, 13. - 4) PPi: papa. 5) $\mathrm{Pl}^{\mathrm{l}}$ : ut (korr. aus aut). $-{ }^{6}$ ) tamen-ecclesiam et om. P. $-{ }^{7}$ ) 2. Mos. 4, 10. 8) E: adheri. - 9) 2 Mos. 4, 15. - 1) E: licent. - 11) E: intentum. - 19) E: exsequitur. -- 13) E: a deo: - 14) Er. Joh. 21, 15. -- 15) PP1: in quit. E: inquit. - 19) E: Eciam. - 17)•E: synodalibus. - 18) E. relata. - 19) E: inmedia, cerninus effectum, quia. -- ${ }^{20}$ ) E: sui effectum. 
inter deum et papam sint medii secandum ordinem suppositorum, quia deus eligit sibi papam per cardinales secundnm ordinem, tamen virtute deas inmediate eligit sibi papam, et confert ei papatam. Eleccionem enim summornm pontificam sibi deas reservavit1), licet ${ }^{2}$ ) eleccionem eorum bonis sacerdotıbus et spiritualibus populis concessit, dicitur distinccione $\mathbf{7 9}$, capitulo altimo, et ideo obieccio nulla. Tercio ${ }^{3}$ ) si servus malus et piger non aliunde dampnatus est, nisi quia contra fidem sibi a deo conmissi talenti, abiens fodit illad in terram et abscondit, Mathei 304). Manifestum est, quod, quicanque facit contra fidem sibi a deo conmissi, ponit se in statum dampnacionis. Sed deas fidei pape conmisit papatum, et ergo. papa, si renuncciaret sine licencia dei papatui, faceret contra fidem sibi a deo conmissi, et poneret se in statam dampnacionis. Et ideo nullo modo papa potest sine licencia dei papatui renuncciare. Quarto cum deus sit, qui Petro dicit, et in eo snis successoribus: tibi dabo claves regni celoram, Mathei $16^{5}$ ), et eleccionem summornm pontificum sibi reservavit, a deo, et non ab homine, anctoritas pape dependet, conservatur et manutenetur in papa. Et ergo papa, cum non poterit potestati dei resistere, non potest sine eins licencia a se papatum abicere et ei renuncciare. Quinto neque propter adversa quecanque vitanda, neque propter lacra quecunque conquirenda papa potest sine licencia dei renunccisre care pastorali ovium sibi conmissarum, quia bonus pastor animam suam dat pro ovibus suis, Jobannis $10^{6}$ ), et a Christo in persona primi pape dictum est sibi preceptive: non fuge, sed pasce oves mess?), et ad hoc pertinet: pasce oves meas, ut ponas animam tuam pro ovibas meis secundum Augustinum super Johanne sermone $47^{8}$ ), et ergo nullo modo papa potest papatni sine licencia dei renuncciare. Et si argueret, non sequitur: aliquando oves meas licite potero fugere, ergo animam meam pro ovibus meis non teneor ${ }^{9}$ ) ponere. Nam qui dixit: cum autem persequentur vos in civitate ista, fugite in alism, Mathei $10^{10}$ ), ecism precepit, quod bonus pastor dat animam suam pro ovibas suis, Johannis 10 . Sexto nullus alius preter deum potest papam deponere, quis cunctos ipse indicatarus a nemine est indicandus, nisi deprehendatur a fide devius, distinccione 40, si papa. Nec ipse se potest destituere sine licencia dei, quia prohibitnm est, quod aliquis sit. sibi index, codice: ne quis in sua causa indicet, ymmo prestancior, cum debeat esse iudicans, quam illa res est, de qua indicatur secundum Augustinum. Inde vera religione implicat contradiccionem, et ergo papa non potest sine licencia dei papatui renuncciare. Septimo non tenet renuncciacio nisi de licencia superioris, cuius est institucio, ut colligitur ex multis capitalis de translacione et renuncciacione; et papa nullum superiorem habet nisi deum de eleccione, licet et a deo instituitur et habet potestatem per supra allegata, et ergo non potest sine licencia dei papatui renuncciare.

1) $\mathrm{E}$ : reservabit. $\left.-{ }^{2}\right) \mathrm{P}$ : et in statt licet.

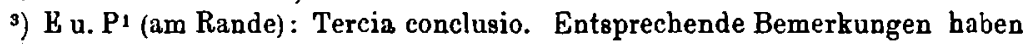
E u. $\mathrm{P}^{1}$ auch bei den spateren Konklusionen.

4) Richtig Matth. 13, 44. - s) Matth. 16, 19. - ') Ev. Joh. 10, 11. 7) Ev. Joh. 21, 15-17. - 8) $\mathrm{P}^{1}:$ 42. - $\left.{ }^{9}\right) \mathrm{P}:$ timeo. - 10) Matth. 10, 23. Die Worte eciam - Johannis om. P. 
Forte ${ }^{1}$ ) dicis: glossa de renuncciacione libro 6, capitulo 1 dicit, quod renancciacio fieri debet in manibus superioris. Fateor id veram, abi est superior, sed hic propter inpossibilitatem id servari non potest. Item ait: inferiores prelati a suis superioribus prelatis confirmantur ${ }^{2}$ ). Unde, inquit, non est mirum, si sine eorum licencia renuncciare non possunt, sed papa a nemine confirmatur. Unde sicut sine superiore instituitur, ita sine superiore ${ }^{3}$ ) renancciat, sed errat et inplicat in terminis, et ergo non allegari, sed reprobari debet. Nam sequitur: renuncciacio fieri debet in manibus superioris, ubi est superior. Sed papa habet superiorem verum, scilicet deum, et verum hominem, cuius est vicarius; et ergo papa non potest renuncciare, nisi in manibus veri dei et veri hominis. Item sic inferiores prelati a sais superioribus prelatis confirmantur et institunntar. Unde, inquit, non est mirum, si sine eorum licencia renuncciare non possunt, sed papa ${ }^{4}$ ) a deo elegitur, distinccione 79 , capitalo nltimo, a deo instituitur, Mathei 16: tibi dabo claves regni celorum, a deo confirmatur, Johannis 21: pasce oves mess, et per iura allegata de indiciis novit distinccione 21. quamvis, et distinccione 22. sacrosancta, et a deo edificatur secundum Cirillum episcopum Alexandrinorum libro 2: thezaurorum ut membra, inquit, maneamus, in capite nostro apostolico, throno ${ }^{5}$ ) Romanorum pontificis, a quo nostrorum est querere, quid credere, et quid tenere debeamus, ipsum venerantes, ipsum rogantes pre omnibus, quoniam ipsius solius est reprehendere, corrigere, statuere et disponere, solvere et ligare loco illius, qui ipsum edificavit, et nulli alii, quod suum est plenum, sed ipsi soli dedit, cui omnes iure divino capnt inclinant, et primates mundi tamquam ipsi domino Jhesu Christo obedinnt; et ergo papa non potest sine licencia dei papatai renuncciare. Si enim inferior prelatus non potest renuncciare sine licencis superioris, a quo instituitar et confirmatar, quanto magis papa, qui a deo eligitur, institaitur, confirmatur et edificatur, non poterit renuncciare sine licencia dei, cui obedire oportet magis quam hominibus, Actunm $5^{6}$ ). - Octavo de translacione, capitulo inter corporalia dicitar: cum ergo forcius sit spirituale vincalum quam carnale, dubitari non debet, quin omnipotens deus spiritnale coniugium? ${ }^{7}$ ), quod est inter episcopum et ecclesiam, suo tantum indicio reservavit dissolvendam; qui dissolucionern et eciam carnalis coningii, quod est inter virum et feminam, suo tantum iudicio servavit precipiens, ut, quos deus coniunxit, homo non separet, Mathei $19^{8}$ ). Sed inter papam et ecclesiam coningium est spiritaale, et ergo omnipotens deus suo tantum iudicio illud reservavit dissolvendum. Papa ergo non potest se ab ecclesia separare sine licencia dei et renuncciare. Et si hanc ipsam licenciam non noverit se habere ex scriptura aut instincta ${ }^{9}$ ) spiritus sancti, quemadmodum Cyriacus et alii sancti summi pontifices ${ }^{10}$ ), qui renuncciaverunt, teneat pro certo, quod non potest papatui renuncciare. Sed utrum instinctu spiritus sancti papa sit licenciatus ad renuncciandum, homo alter ab eo non potest

2) E am Rande bei Forte: Obieccio. - $\left.{ }^{2}\right) \mathrm{P}^{1}$ : et instituuntur. - ${ }^{\text {s) }}$ insti. tuitur - superiore om. P. - ") papa om. P. - 5) E P1: trono. - 6) A postelg 5, 29. - 7) $\mathrm{P}$ : convivium. - s) Matth. 19,6. - 9) E: in distinctu.

10) u. a. Cölestin V. im Jabre 1294. Seine massgebliche Bulle datierte rom 13. Dezember 1294. Über Cyriacus, oben S. 426. 
cognoscere. Quis enim scit hominam, que hoıninis sunt, nisi spiritus hominis, qui in ipso est? 1. Corinthioram 2 ${ }^{1}$. Et ideo, ne eis, qui scripturam non sciunt, evidencia premisse conclusionis papa presumat dicere, quod eciam pro indurato scismate tollendo non valeat renuncciare, et quod, si renuncciaret, verbotenus adbuc remaneret verus papa, ideo ex sacris eloquiis sit conclusio tercia illa: dei licencia est, quod papa pro induratio scismate tollendo renuncciet. Nam cam sit manifestum, quod deus pape conmisit papatum ad edificacionem ecclesie, et non destruccionem, 2. Corinthiorum 10 et $13^{2}$, manifestam est, quod factum pape, quocienscunque edificacio fuerit ecclesie, de licencia dei est, sed si pro indurato scismate tollendo renuncciaret, edificacio foret ${ }^{3}$ ) ecclesie, quia infallibiliter eius sequeretur unio et reintegracio, et ergo dei licencia est, quod papa pro indurato scismate tollendo renuncciet. Secundo dei licencia est, quocienscunque papa bona conmunia propriis anteponit, quia illud, secundum Augustinum in regula, est opus caritatis, que diffasa est in cordibus nostris per spiritum sanctum, ad Romanos $5^{4}$ ), et maior quam in ceteris Christianis debet esse in papa, 24. questione 1. officii. Sed si pro indurat, scismate tollendo renuncciaret, bona conmunia ecclesie bonis propriis anteponeret, et ergo dei licencia est, quod pro indurato scismate tollen:10 renuncciet. - Tercio si papa pro indurato scismate tollendo renuncciaret, quereret non, quod sibi utile est, sed quod inutile. Sed semper dei licencia est, quando papa querit, non quod sibi utile est, sed $^{5}$ ) quod multis, - 1. Corinthioram $10^{6}$ ): non querens, quod wichi utile est, sed quod multis; - et ergo dei licencia est, quod papa pro indurato scismate tollendo renuncciet. Quarto racio institutionis pape a deo tota est: pascere oves Christi Johannis 21. Et ergo in pascendis ovibus Christi necessitas, quando inpellit, quod papa renuncciet, dei licencia est, quod renuncciet. Sed stante indurato scismate necessitas in pascendis ovibus Cbristi impellit, quod papa renuncciet?) eo, quod ad pastorem verum, a quo pascende sunt oves Christi abiecte ${ }^{8}$ ), nisi papa renunceiet, inde possunt ${ }^{9}$ ), aut saltem non speratur, quod possunt reduci; et ergo dei licencia est, quod papa pro indurato scismate tollendo renuncciet. Quinto: finis ${ }^{10}$ ) enim papatus cum sit dirigere universalem ecclesiam ad felicitatem eternam, et deus non velit ad inpossibile aliquem obligari, ut innuit Jeronimus in exposicione katholice fidei, dei licencia est, quod papa, quando non sufficit dirigere universalem ecclesiam ad felicitatem eternam, renuncciet, ut ait decretale de renuncciacione libro 6. supra allegata. Sed stante indurato scismate papa, cam non sufficiat mediam quasi partem ecclesie de manu eripere furis perditoris ${ }^{11}$ ), non sufficit dirigere universalem ecclesiam ad felicitatem eternam. Et ergo dei licencia est, quod papa pro indurato scismate tollendo renuncciet. Sexto, cum enim de licencia dei fiat omne, quod fini ordinacionis eius congrnerit, et quod ${ }^{12}$ ) deus regimen pape ad bonum ordinaverit ecclesiastice unitatis, manifestum est, quod,

1) 1. Korinth. 2, 11. - 2) 2. Eorinth. 10, 8 u. 13, 10. $-{ }^{3}$ ) E: fuerit. 4) Röm. 5, 5. - 5) quad inutile - sed om. P. $-{ }^{6}$ ) 1. Korinth. 10, 33. - i) Sed stante - renuncciet om. E. $-{ }^{8}$ ) E: abiecta. $-{ }^{9}$ ) inde possunt om. E. $-{ }^{10}$ ) d. i. Zweck. - 11) E: finis proditoris. - ${ }^{12}$ ) quod om. E; $\mathrm{P}^{1}$ : durchstrichenes ergo (statt quod). 
quanto congruencius papa efficeret bonum ecclesiastice onitatis, tanto licencins per deum ageret. Sed congruenter efficeret bonnm ecclesiastice unitatis, si pro indurato scismate tollendo renuncciaret. Et ergo dei licencia est, quod papa pro indurato scismate tollendo renuncciet. Septimo, cum enim dominus, utrum se diligeret, Petrum, prius quam ei ecclesiam conmiserat, interrogaret, Johannis 21., manifestam est, quod papa, qui successor est Petri non nisi secnndum legem caritatis, debet officium pastorale assumere et ecclesiam regere. Sed caritas anitiva est, et non divisiva 1), cum sit vis unitiva et concretiva, ut ait Dyonisius 4. capitulo de divinis nominibus ecclesie: unitiva est et non divisiva; et ergo papa, quod in se est, ex debito officii pastoralis et secundum legem caritatis tenetur facere, ut ecclesia uniatur, et induratum scisma tollatur. Sed si renuncciaret, ecclesia uniretar, et indaratum scisma tolleretur, ergo papa ex debito officii pastoralis, et secundum legem caritatis, pro indurato scismate tollendo tenetur renuncciare. Omne autem opus conforme legi caritatis de licencia dei est, ergo ${ }^{2}$ ) dei licencia est, quod papa pro indurato scismate tollendo renuncciet. Et inde sequitur conclusio quarta: qui se papam dicit Gregorium 12., notorins votifractor effectus est et periuras, nam universo orbi hoc verbum notum est, quod spe et hac $^{3}$ ) intencione (ardinsles Gregorium ${ }^{4}$ ) elegerunt et assumpserunt in episcopum animarum nostrarum et universalis ecclesie, et gregis dominici pastorem, quod infra spacium, unius scilicet anni et trium mensinm, adepto papatai pro indurato scismate tollendo renuncciaret, et daret pacem ${ }^{5}$ ) ecclesie. Et hoc ipsum in conclavi ${ }^{6}$ ) et in primo suo consistorio ipsemet promisit, vovit et iuravit; et quia dei licencia est, quod papa pro indurato scismate tollendo rentuncciet, et ergo promissum eius est laudabile, votum pium, et iuramentum licitum, et de iure non solnm divino, sed eciam naturali tenebatur ea termino diffinito adimplere. Sed heu cupidus bonoris temporalis magis quam eterni, et suam utilitatem, et non que ?) gregis est dominici querens salutem, noluit iuramentum ${ }^{8}$ ) adimplere, neque votum observare, quando tamen illad potait adimplere et istud observare, quia per procuratorem potuit cedere et eciam in manibus sui collegii renuncciare; et ergo notorius votifractor effectus est et periuras. Nec est verum, ut hostis sancte unionis ferventissimus ille uunc Ragusinus, cuins memoria in malediccione est $^{9}$ ), per omnes ecclesias in sua epistola occulte innuit, quod Gregorjus in melius inramenta, vota et promissiones secundum divina ${ }^{10}$ ) iura conmutavit. Non enim locum conmutacio iaramenti aut voti in melius babet, nisi quando aliquid melius faciendum occurrit ad conmunem atilitatem. Sed nichil faciendum melius est bono unitatis ecclesie, quod bonum est 11) convenientissimum et ideo divinissimum, 1. Ethicorum; et ergo Gregorius iuramentum aut rotum non potuit neque potest in melius conmutare, turn propter rem iuratam, tinm quia nemo in propria causa potest

1) unitiva - divisiva om. PP'. - 2) EP': et ergo. $\rightarrow$ s) P : ac intencione. 5) E: Gregorium 12. - 5) E: potestatem. - 5) P: conclario. - 7) E: qui. s: querens-iuramentum om. P.

9) Falkenberg hatte, wie die obigen Worte ergeben, von dem am 13. Januar 1408 erfolgten Tode Nikolaus' schon Nachricht erhalten. - $\mathrm{P}^{1}$ : Rakusinus.

10) $\mathrm{E}$ : dominica. - 11) $\mathrm{P}$ : et. 
fieri iudex, per allegata. Et inde sequitar conclusio quinta: Gregorius pusilloram scandalizator est maximas, nam si ordinacio orbis conponitur ${ }^{1}$, principis ad exemplam, nec sic inflectere sensus bumanos edicta valent, at vita principis inxta illud Clandiani in maiori: mobile mutatur semper cum principe valgas. Et Gregorius erat princeps, cuius vitam ${ }^{2}$ ) omnium mortalium oculi, omnium iudicia, omniumque vota considerant. Quia quanto erat super ceteros homines in loco excelsiori, tanto omninm patuit aspectibus luce clariori. Non enim potest civitas abscondi supra montem posita, inquit salvator, Mathei $5^{3}$ ). Et ergo cum sit traditus in reprobum sensum, ut recuset, quod laudabiliter promisit solvere, refugiat, quod rite vovit, reddere, contempnat, quod licite iuravit, adimplere, manifestum est, quod subiecti quique non tam ${ }^{4}$ ) verba eius, que audiunt, sectantur, quam sola, que conspiciunt pravitatis ${ }^{5}$ ) exempla, imitantur. Et ideo pusillorum scandalizator est maximus, et iuxta sentenciam veritatis Mathei $18^{6}$ ), expedit ei, ut suspendatur mola asinaria in collo eius, et demergatur in profundum maris. Et si papa, quando sue et fraterne salutis negligens deprehenditur, inatilis et remissus in operibus suis, et insuper a bono taciturus, quod magis officit sibi et omnibus, nichilominus innumerabiles populos catervatim secum ducit, primo mancipio gehenne ${ }^{7}$ ) cum ipso populo plagis multis vapulaturus in eternum, distinccione 40 , si papa; quanto magis Gregorius, [quoniam] innumerabiles populos catervatim secum ducit, plagis multis vapulaturus in eternum, qui declinat a bono et est taciturus, et palam facit malum. Conclusio sexta: Gregorius nutritor indurati est scismatis. Hoc licet sit verum, quia scindit ecclesiam, que scindi non debet, in cuius signum milites de tunica inconsutili, Johannis $19 .{ }^{8}$ ), dixerunt: non scindamus eam, sed sorciamur de ea, cuius sit, tamen per opera eius sensui manifesta $^{9}$ ) evidencius est probandum. Dicit namque beatus Thomas 2. secunde, questione 39, articulo 1, quod scismatici proprie dicuntur, qui sponte et propria intencione se ab unitate ecclesie separant. Unitas autem ecclesie in duobus attenditur, scilicet in conmixcione membrorum ecclesie adinvicem seu conmunicacione, et iterum in ordine omnium membrorum ecclesie ad unum caput secundum illud ad Collocenses $2^{10}$ ): Inflatus sensu carnis sue, et non tenens caput, ex quo totum corpus per nexus et coniuncciones subministratum et constructum, crescit in augmentum ${ }^{11}$ ) dei. Sed Gregorius sponte et propria intencione se ab unitate ecclesie separavit, quia ambobus collegiis et omnibus utriusque obediencie regibus, principibus, prelatis, universitatibus, connunitatibus et ceteris, quorum interest, in unum propositum et roluntatem dandi pacem populo Christi et extirpandi scisma antiquitatum per eleccionem unici et indubitati pastoris universalis ecclesie iunctis, ipse ${ }^{12}$ ), quamvis ab hiis omnibus aut eorum ambasiatoribus pro hac causa in curia degentibus sepissime sit requisitus, et monitus per inmensam miseriam dei, ut memor finis pastoralis officii, quod in salutem dirigitur animarum, ac iuramenti et roti, que tam solempniter prestita $a b$ es et toti mundo publicata fuere. dignetur ${ }^{13}$ ) unionem populo

1) E: ponitur. - 2) E: vita. - s) Matth. 5, 14. - 4) E: tamen verbo. 5) E: gravitatis. - $\left.{ }^{6}\right)$ Matth. 18, 6. - 7 ) E: Jehenne. - 8) Ev. Joh. 19, 24. ๑) E: manifestat. - 19) Koloss. 2, 18-19. - 11) E: augmentacionem. - 19) E: si jpse. - 19) E: dignaretur. 
Christi accelerare et dare pacem per viam cessionis electam, que iusta est, et quam ante iarando et rovendo iustam censuit, omnium salubria monita contempsit, quod' ${ }^{1}$ via cessionis et renunccionis est dyabolica et dampnata, asseruit, et quod illam amplius non erat intencionis prosequendi, ad nonnallos ${ }^{2}$ ) litteras destinavit, cardinalibus sub pena privacionis cardinalatus, periurii et beneficiorum oretenus inhibuit, ne cum ambasiatoribas Benedicti de Avinione $^{3}$ ) vel regis Francie de materia unionis amplius loquerentur, et quod sine suo mandato de cetero non congregarentur. Et breviter, quanto plus monitus, ut se membris ecclesie in ordine ad unicum et indubitatum caput comisceret, tanto plus obtusis auribus induratum se prebuit, et ergo scienti nomen scismatici4) evidens est, quod verus est scismaticus et nutritor buius indurati scismatis. Et si ex signo secundo priorum licitum sit arguere, signum huius evidentissimum est, quod magistrum in sacra theologia ideo, quia in publico sermone ${ }^{5}$ ) exhortatus est eum, at iuxta iuramentum suum et votum, que gentes divine eciam legis ignare naturaliter observant, et enunciaret6) et scisma tolleret, in carcerem religavit et postea nullum, nisi prius eius sermone perlecto, et securatus, quod de materia unionis nichil dissereret?), permisit coram se predicare. Septima conclusio subditi: quique tenentur Gregorio obedienciam subtrahere. Cum enim obediencia prelato debeatur et ex ordine prelacionis ${ }^{8}$ ) causetur, ad illa tantummodo debitum obediencie se extendit, et ex debito obediencie subditus obligatur, ad que ius ${ }^{9}$ ) prelacionis se extendit et est ordinatum. Sed constat, quod ins prelacionis datum est pape non in destruccionem, sed in ecclesie edificacionem, 2. Corinthiornm 10 et $13^{10}$ ). Et Gregorius nititar non solum destruere, sed eciam destruit ecclesiam, quia dividit eam et manutenet scisma antiquatum. Et ergo debemus sibi resistere et non obedire, sic enim precepit deus, ut in malis nulli potestati obedismus. Secundo subditi, qui possunt, tenentur Gregorio in faciem resistere, et ergo a multo forciori obedienciam subtrabere. Nam si Paulus Petro, qui papa erat apostolorum, propter inminens periculum circa fidem in faciem coram omnibus resistit, ad Gallathas $2^{11}$ ), quilibet nunc, qui potest, tenetar Gregorio nutrienti scisma antiquatum, descendens in heresim, in faciem coram omnibus resistere. Et qui non resistit, errorem snum et facinus convincitur approbare. Error enim, cui non resistitur, approbatur, nec caret scrupulo societatis occulte, qui manifesto facinori desinit obviare, distinccione 83 , error. Et in capitulo constitutis primo extra de testibus. Tercio, cum enim ex dictis sit evidens, quod Gregorius papatum occupat contra proprium iuramentum et manatenet scisma antiquatum, evidens eciam est, quod obedire Gregorio at pape, est ipsum fovere in periurio, et manutenere scisma antiquatum. Sed omnis, qui fovet ipsum in periurio, et manutenet scisma antiquatum, peccat mortaliter et est in statu eterne dampnacionis; et ergo omnis, qui obedit 12) Gregorio ut pape, peccat mortaliter et est in statu dampnacionis perpetue, ymmo fantor heretice pravitatis, et si ei pertinaciter persistit

1) E: quia. - 2) E: a domino nullas. -- s) d. i. Benedikt XIII (Pedro de Luna). - t) P u. E om. scismat:ci. - 5) E om. sermone. - 6) E : renuncciare. 7) $\mathrm{E}$ : diceret. - 8 E: prelatorum. - 9 ) E: quevis. - io) E (unrichtig) : 14. 11) Gal. 2, $7 \mathrm{ff.} \mathrm{-} \mathrm{12)} \mathrm{Im} \mathrm{Text} \mathrm{von} \mathrm{E}$ ist hinter obedit längere Lücke. 
adherere, efficitur bereticus. Recedite ergo, reges et principes, conmunitates et universitates, ville el civitates, castra et opida, pasilli et magni, mares et fomine, omnes et singuli, temporales et spirituales, ab obediencia imperii Gregorii, qui multos secum trahens decipit, contempnens sacerdotes Christi, cardinales, et se a clero plebisque societate segregans construere andet sibi novam synagogam! Practica huius conclusionis observata est, distinccione 19, Anastasius, quando temporibus Theodrici regis multi clerici se a conmunione Anastasii, ideo quia inciderat in heresim iam dampnatam, abegerunt. Et similiter 16. questione ultima, capitulo sane, quando quadraginta episcopi a proprio metropolitano, qui eciam inciderat in heresim iam dampnatam, discesserant. Veritati tamen huius conclusionis quidam resistunt et sic argaunt: elericis ante tempus sentencie ab episcopo suo discedere non lieet, 8. questione 4, nomiue. Sed Gregorius nondum est sentenciatus, et ergo clericis ab eius obediencia non licet discedere. Sed errant, nam licet in aliis criminibus clericis ante sentenciam ab episcopo suo discedere non liceat, in crimine tamen heresis licet per iura allegata et 24. questione prima, capitulo 1: vel respondendo ad verba dicendum, quod in omnem, qui incidit in heresim iam dampnatam, sentencia est lata canonis 24 questione 1, Achacius. Et quia Gregorius incidit in heresim iam dampnatam, quia natritor est scismatis antiquati, quod in heresim descendit, et ergo in ipsum est sentencia lata canonis. Et nullus ab eius obediencia ante sentenciam discedit. Octava conclusio: Gregorins data eius pertinacia hereticus est censendus. Nam certum est, quod omnis, qui pertinaciter nutrit heresim et sustinet, hereticus est censendus, ut protat diffinicio heretici, quam ponit Augustinus is libro de civitate dei, capitulo 51: qui, inquit, in ecclesia Christi morbidum aliquid pravumque sapinnt, si correpti, ut sanum rectumque resistunt contumáciter, suaque pestifera et mortifera docmata ${ }^{1}$ ) emendare nolunt, sed defendere persistunt, heretici fiunt, et foras exeuntes babentur in exercentibus inimicicias eciam, si quippe veris illis katholicis membris malo suo prosunt, dam deus utitur et malis bene, et diligentibus omnia cooperantur in bonum. Sed omnis, qui pertinaciter induratum nutrit scisma, pertinaciter nutrit heresim et sustinet, quia scisma, licet in principio aliqua ex parte intelligi potest diversum ab heresi, tamen, quia est via ad heresim, eius indaracione et obstinacione in fine descendit in heresim, et nullum scisma est, nisi aliquam beresim confingat, ut recte ab ecclesia recessisse videatur, ut ait sanctus Jeronimus in epistola ad Gallathas, et transsumitur 24. questione 3, inter scisma. Et ergo omnis, qui pertinaciter induratum nutrit scisma, hereticus est censendus. Sed Gregorius induratum nutrit scisma ex conclusione 6, et ergo data eius pertinacia bereticus est censendus. Ft hec pertinacia revincenda est ex rebellinne post trinam monicionem, 24. questione 3, de illicita. Et superhabundanter post quartam: quia qui ter argutus non obedivit, potest in quarto obedire, ait Jeronimus exponens illud Mathei $18^{2}$ ): sit tibi sicnt ethnicus; secundo doctor sanctus ${ }^{3}$ ) secunda secunde, questione 5. articalo 3. dicit, quod formale obiectum fidei est veritas prima, secundum quod manifestatur in scripturis sacris et doctrina ecclesie. Onde ait: quicunque non

1) Statt dogmata. - 2) Matth. 18, 17. - s) Thomas von Aquino. 
inheret sicut infallibili et divine regule ${ }^{1}$ ) doctrine ecclesie, que procedit ${ }^{2}$ ) ex veritate prima in scripturis sacris manifesta, ille non habet habitum fidei. Sed ea, que sant fidei, alio modo tenet quam per fidem. Sicat si aliquis teneat mente aliquam conclusionem non cognoscens medium illius demonstracionis, manifestum est, quod non habet eius scienciam sed opinionem solum. Jlanifestum est autem, quod ille, qui inheret doctrine ecclesie, tamquan infallibili regule, omnibus assentit, que ecclesia docet, alioquin si de hiis, que ecclesis docet, que valt, tenet, et que vult, non tenet, non iam inberet ecclesje doctrine sicut infallibili regule, sed proprie voluntati. Sed Gregorius non inberet sicat infallibili et divine regule doctrine ecclesie. Si enim inhereret doctrine ecclesie tamquam infallibili regnle, omnibus assentiret, que ecclesia docet; sed iam non omnibus assentit, que ecclesia docet. Ecclesia enim docet, quod Romanus pontifex potest libere resignare, de renuncciacione capitulo 1 , libro 6 . Et huic non assentit, sed oppositum asserit, quia dicit, quod via cessionis et renuncciacionis est dyabolica et dampnata. Et ergo manifestum est, quod non habet habitum fidei, et quia prius habuit vel saltem putabatur babere habitam fidei, et ideo cum omnis talis sit hereticas censendus, qui fuerit pertinax, manifestum est, quod Gregorius data eius pertinacia hereticus est censendus. Et ideo cam omnis homo simili sibi sociabitur, Ecclesiastici $13^{3}$ ), sectenarios et fraticellos, undecunque veniunt, de heresi suspectissimos effectu singularis amoris prosequitur et promovet, ad alta eorum aquiescens consiliis, aliis, qui ecclesiam edificare dei in doctrina sana sunt ydonei, avulsis. Tercio ${ }^{4}$ ), quicunque pertinaciter negat ecclesiasticam unitatem, contra articulum fidei ,Unam sanctam6, hereticus est censendus. Sed Gregorius negat ecclesiasticam unitatem contra articulum fidei ,Unarm sanctam', quia scindit ecclesiam et manutenet scisma antiquatum, et ergo data eius pertinacia hereticus est censendus. Nec impedit, quod ore non negat ecclesiasticam unitatem, quia negacio non est ${ }^{5}$ ) tantum lingua, sed eciam fit et factis, ad Thytum 16): confitentur se nosse deum, factis aulem negant. Ft glosa ibi dicit: quisquis factis negat Christum, antichristus est. Tales sunt omnes mali katholici, qui non verbis sed factis negant. Cum ergo negacio factorum maior sit quam verboram, quis opera persuadenciora sunt sermonibus, 10. Ethicoram, et plus est facere facto quam verbo, capitulo dilecti de appellacionibus, Gregorius, licet non lingua, tamen facto et plus quam lingus ecclesiasticam negat nnitatem. - Conclusio nona: Gregorius data eins pertinacia de iure papatu est privatus. Nam certum est, quod omnis hereticus de iure honore sno et officio est privatus, 7 . questione 1, Novacianus, 24. questione 1: dicimus de prescripcionibus capitulo 1 , et de hereticis ad abolendum. Sed Gregorius data eius pertinacia hereticus est censendus per conclusionem octavam. Et

1) et om. Pi. - 2) P! : procedunt. - 3) Eccl. 13, 20.

i) Von hier an wieder in $E$ (Seite 343 ) vorhanden; dagegen fehlt in $\mathrm{P}^{\prime}$ hinter avulsis der Rest des Traktats. Die von abweichender, etwas späterer Hand des 15. Jahrhunderts geschrievenen Explicitworte in P1 lauten:, Istam questionem suprascriptam Ad pauca respicientes etc. determinarit dictus de Walkem. berg Prage anno domini 1408, proxima feria sexta post festum sancti Nicolai •.

5) est om. E. - 6) -Titus 1, 16. 
ergo data eius pertinacia honore suo et officio, et per consequens papata, de inre est privatus. Secundo omnes heretici, cum sint extra ecclesiam, quia foras exeuntes habentur in exercentibus inimicis, non habent aliquam potestatem in ecclesia, sed Gregorius data eius pertinacis non habet aliquam potestatem, et ideo nec papatum in ecclesia. Tercio Ambrosins in libro de Paradiso dicit, quod, qui solvendi ius non habet, nec ligandi habet. Et infra: certum, inquit, est. quod ecclesie atrumque licet, heresis ntrumque non habet. Sed Gregorius data eius pertinacia hereticus est censendus, et ergo data eius pertinacia neque potestatem habet solvendi neque ligandi, et per consequens neque papatum, cuius proprius est actus solvere et ligare, Mathei $16^{1}$ ). - Conclusio decima: data pertinacia Gregorii cardinales possunt procedere ad eleccionem unici et indubitati pastoris universalis ecclesie. Nam demonstratum est, quod Gregorius papa data ${ }^{2}$ ) eius pertinacia de iure papatu est privatus, et sedes vacat. Sed vacante sede cardinales possunt procedere ad eleccionem unici et indubitati pastoris universalis ecclesie. Et ergo eciam data pertinacia Gregorii possunt procedere ad eleccionem unici et indubitati pastoris universalis ecclesie. Et huius rei gracis possunt, - ut docet capitulum, nunc autem, distinccione 21, ecclesiam convocare et Gregorium vocare atque requirere, at tollat scisma via iurata. Quam si recusare perseveraverit, crescente eius pertinacia et perdurante, debent iuxta capitulum, si papa, distinccione 40, absentem sive presentem iudicare, et non obstante eius absencia ad eleccionem unici et indubitati pastoris universalis ecclesie procedere; nec timere debent penas, quas forte minatur, aut privaciones. Si enim omne, quod ab hereticis actum fuerit, initum est et omnino cassandum, 12. questione 2, alie naciones, 25. questione 1, omne, et extra de scismaticis capitulo 1 , et Gregorius data eius pertinacia bereticas est censendus. Omne, quod ab eo, data eius pertinacia, actum est vel fuerit, debet omnino cassari, nec potuit cardinales novos creare, nec eciam antiquos privare. Et ergo trepidare timore non debent cardinales, ubi timor nullus est, sed confisi in eo, cuius res agitur, modo ostenso viriliter, ne amaritudinem eterne dampnacionis ex negligencia incurrant, inceptum debent perficere et extirpare scisma antiquatum per eleccionem unici et indubitati pastoris, successoris Petri et vicarii veri dei et reri hominis Jhesu Christi, qui semper benedictus est et gloriosus in secula seculorum amen."

In Kodex E, S. 344 schliesseu sich folgende Verse an, die wohl nicht ron Falkenberg, soudern ron dem bald nach 1410 arbeitenden Kopistèn herstammen:

,Gregorius, vix catholicus, papa duodenus,

Ad baratrum tendit, Petri bona recia vendit,

Vovit, iuravit, promisit, ratificavit,

Heu modo periurans, vota non solvere curans.

0 miser Gregorius! nescis horam moriendi.

Cur non scisma leves, cum dies sint tibi breves?

$\mathrm{Ne}$ diffamaris, tolle scisma, sicque bearis! ${ }^{3}$ )

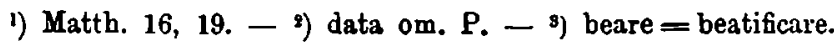


Johannes Ragusinus ${ }^{1}$, frater Gabriel Gabbadeus ${ }^{2}$ ),

Quatuor equales inutiles carpatinales ${ }^{3}$ ),

Heinricus Rodens Tudertinus ${ }^{4}$, male videns,

Consors Laudensis ${ }^{5}$ ), Christi quos indicet ensis.

Sunt ypocrite, fraticelli, sodomite,

Nequam latrones, proch periurii buzerini6)!

Christum deludant, cum papa sathana ludunt,

Simul priventur, ad ignem proicientur."

i) Johann Dominici war am 12. Mai 1408 zum Kardinal erhoben worden. Der Satansbrief eines Ungenannten zu Siena war kurz vorher gegen ihn veröffentlicht worden: Erler S. 24; J. Lenfant, Histoire du concile de Pise. Bd. I. Amster. dam 1724, S. 197; M. Souchon, Die Papstwahlen in der Zejt des grossen. Schismas, 1378-1417. Bd. I. Brannschweig 1898. S. 154.

2) Vielleicht identisch mit Gabriel Condulmaro? Dieser war ebenfalls am. 12. Mai 1408 zum Kardinal kreiert worden, vgl. Röвler S. 160.

b) Von carpatinus abgeleitet.

4) Aus Todi.

5) Der zur Partei der Pisaner Kardinäle gehörige Jacobus Balardi, Bischof von Lodi, 1407-1417; erwähnt u. a. bei F. Bliemetzrieder, Das Generalkonzil im grossen abendlăndischen Schisma. Paderborn 1904. S. 279, Anm. 5.

b) Statt bucerini (ron bos). 